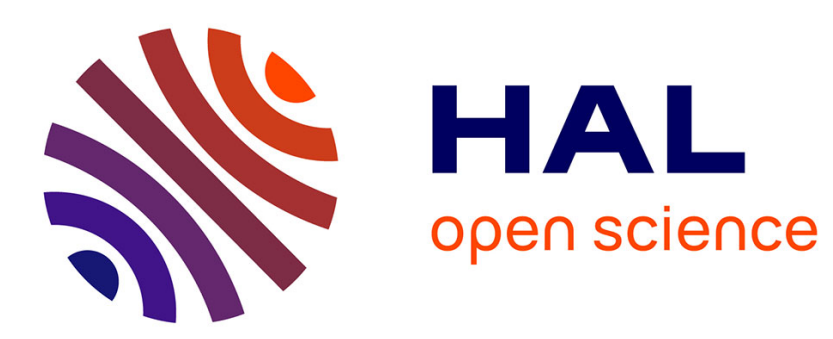

\title{
Effect of Surface Tension Variations on the Pinch-Off Behavior of Small Fluid Drops in the Presence of Surfactants
}

\author{
Matthieu Roché, Mounir Aytouna, Daniel Bonn, Hamid Kellay
}

\section{To cite this version:}

Matthieu Roché, Mounir Aytouna, Daniel Bonn, Hamid Kellay. Effect of Surface Tension Variations on the Pinch-Off Behavior of Small Fluid Drops in the Presence of Surfactants. Physical Review Letters, 2009, 103 (26), pp.264501. 10.1103/PhysRevLett.103.264501 . hal-00670463

\section{HAL Id: hal-00670463 https://hal.science/hal-00670463}

Submitted on 20 Dec 2017

HAL is a multi-disciplinary open access archive for the deposit and dissemination of scientific research documents, whether they are published or not. The documents may come from teaching and research institutions in France or abroad, or from public or private research centers.
L'archive ouverte pluridisciplinaire HAL, est destinée au dépôt et à la diffusion de documents scientifiques de niveau recherche, publiés ou non, émanant des établissements d'enseignement et de recherche français ou étrangers, des laboratoires publics ou privés. 


\title{
Effect of Surface Tension Variations on the Pinch-Off Behavior of Small Fluid Drops in the Presence of Surfactants
}

\author{
M. Roché, ${ }^{1}$ M. Aytouna, ${ }^{2}$ D. Bonn, ${ }^{2,3}$ and H. Kellay ${ }^{1}$ \\ ${ }^{1}$ Université Bordeaux 1, Centre de Physique Moléculaire Optique et Hertzienne, UMR 5798 CNRS, \\ 351 cours de la Libération, 33405 Talence, France \\ ${ }^{2}$ WZI, University of Amsterdam, 1018XE Amsterdam, The Netherlands \\ ${ }^{3}$ LPS, ENS, 24 rue Lhomond, 75231 Paris, France \\ (Received 15 April 2009; published 22 December 2009)
}

It is shown experimentally that surfactants can change the thinning rate of fluid necks undergoing rupture. In the case of two-fluid pinch-off, two or three linear regimes are observed for the variation of the neck radius versus time. The surface tension in the neck region changes with time, as a result of surfactant depletion. Similar results are obtained for the case of a single fluid pinching in air. The depletion of surfactant can be either partial or complete depending on the rate of transport of the surfactant from the bulk to the surface.

DOI: 10.1103/PhysRevLett.103.264501

Drop formation is a widespread phenomenon which is still incompletely understood. A number of industrial and household processes such as the formation of emulsions, inkjet printing, or the dispensing of liquid drops, involve atomization of liquids in which droplets of a preferred size need to be produced [1]. From the fundamental point of view, the formation of drops implies the rupture of a fluid neck which is a classical problem in nonlinear physics. The latest instances of this process are universal and the solutions to either the liquid neck profile or to the law governing the thinning of the neck display remarkable self similarity and scaling behavior [1,2]. While many experimental studies have focused on drop formation under a variety of conditions [2], none have tackled the issue when surface active agents are present. One may expect the properties of the rupture of the fluid necks to be modified in the presence of agents that can affect the capillary, the rheological, and the elastic properties of the interface between the two fluids. Theory [3] predicts that the asymptotic break up dynamics remain unaltered. Numerical simulations [4-8] however, indicate that profound changes may occur near break up. Here we show that surfactants introduce a dynamic surface tension in the rupture problem leading to changes of the thinning velocity of the neck. This dynamic surface tension arises as the surfactant is depleted from the pinch region due to the stretching of the fluid neck.

We study the two canonical situations: drop formation in another liquid (relevant for emulsification) and drop detachment in air (relevant for spraying). In both cases, the rupturing fluid is chosen sufficiently viscous so that the thinning of the neck proceeds linearly versus time with a thinning velocity proportional to the ratio of surface tension $\gamma$ and viscosity $\eta$.

The first system chosen to produce droplets is a microfluidic chip (Fig. 1) in the so called "flow focusing" configuration [9]. This device allows us to inject, at different flow rates controlled by three syringe pumps, the
PACS numbers: 47.20.Dr, 47.20.Gv, 47.57.Ng, 82.70.Uv

continuous liquid phase through the two side channels and the liquid phase to be dispersed through the central channel. The rupture of the central fluid column occurs near the junction between the three channels in the constriction region. The rupture of the drops is visualized using a microscope and a fast camera working at frame rates up to 50000 frames/s. The liquids used are a liquid crystal (LC: 5CB) which is in the nematic state for temperatures between 21 and $35^{\circ} \mathrm{C}$ [10] and water with surfactant (SDS: Sodium Dodecyl Sulfate) at different concentrations [11]. An advantage of using the LC, despite its complex rheological properties, is that the surfactant is not soluble in the LC so it is present only in the low viscosity outer phase. The use of such a combination of liquids allows us to obtain droplets of a nematic liquid crystal in water [12]. The critical micellar concentration (cmc) of SDS in the LC-water-surfactant system is found to be $8.2 \mathrm{mM}$. The viscosity of the water with surfactant solution is roughly constant versus concentration and close to that of water, $0.87 \mathrm{mPa} \cdot \mathrm{s}$. The temperature was $27^{\circ} \mathrm{C}$.

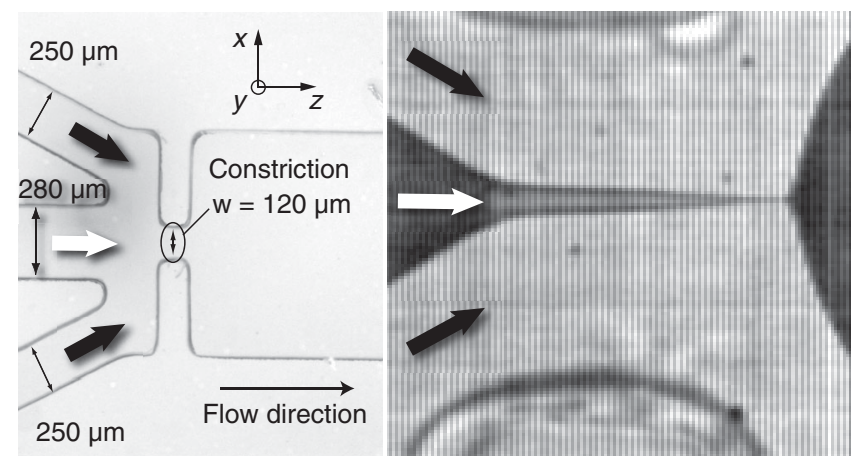

FIG. 1. Schematic of the microfluidic chip. The water solutions are injected along the two side channels (black arrows); the liquid crystal is injected via the middle channel (white arrow). The height of the channel (in the $y$ direction) is $150 \mu \mathrm{m}$. The photo shows the rupture of a drop. 
The viscosity of the liquid crystal depends on the relative direction between the director of the LC orientation and the direction of the shear $[13,14]$. In our case, and in agreement with previous observations [11], the anchoring of the liquid crystal is perpendicular to the interface in the presence of SDS as we have checked through polarizing microscopy observations. These observations show that the LC in the neck during the rupture of a drop is devoid of defects in the last instances before rupture and that the orientation is perpendicular to the interface. No effect of the flow of the outer phase or of the concentration of surfactant on the orientation of the LC is observed. While most of our experiments use a fixed flux for the injection of the LC, variation of this flux from 20 to $600 \mu \mathrm{l} / \mathrm{h}$ (a flux slightly below that of the dripping to jetting transition) did not cause significant variation of the LC pattern. The LC in the neck region is therefore oriented perpendicular to the shear direction for the whole duration of the rupture studied here with a viscosity of $80 \mathrm{mPa} \cdot \mathrm{s}[15,16]$.

The results for the rupture of the liquid crystal in water with SDS, at a concentration of $2 \mathrm{cmc}$ for which the surface is fully covered by surfactant with sufficient amounts of it in the bulk water phase, are shown in Fig. 2. The profile of the neck as well as the minimum neck radius were obtained from photographs similar to the ones shown in Fig. 2. The use of high magnification objectives (50X) allowed us to have excellent spatial resolution down to a fraction of a micrometer. Considering that we are studying a two-fluid pinch-off situation using a large viscosity fluid as the liquid to be dispersed, we expect the thinning to be governed by solutions valid in the so called "viscous" regime [17-20]. The thinning of the minimum neck radius in this case follows the scaling law $h_{\min }(t)=V\left(t-t_{c}\right)$ where $t_{c}$ is the instant of time at which the neck thickness becomes zero. The thinning velocity for a combination of fluids with viscosities $\eta_{1}$ for the outer fluid and $\eta_{2}$ for the inner fluid with an interfacial tension $\gamma$ is given by $V=\mathcal{H} \gamma / \eta_{1}$ where $\mathcal{H}$ depends on the ratio $\eta_{2} / \eta_{1}$. For the viscosity ratios used here the value of $\mathcal{H}$ is roughly $10^{-3}$.

Our main finding is that instead of a constant thinning velocity, the addition of surfactant gives rise to different linear regimes with different thinning velocities. Either 2 or 3 successive linear regimes are observed (Fig. 2) depending on the injection rate of the water solution. Typically we obtained a succession of two regimes for outer fluid fluxes higher than $3 \mathrm{ml} / \mathrm{h}$ (or equivalently a velocity of $4.5 \mathrm{~cm} / \mathrm{s}$ at the constriction) and three regimes below. For much higher rates $(6 \mathrm{ml} / \mathrm{h}$ and higher $)$ we occasionally observed a succession of three regimes with the third regime lasting only a very short time and the rupture occurring rather near the injection than near the drop. In both cases, the thinning starts out roughly linear and accelerates as the rupture is approached. When 3 linear regimes are present, the thinning decelerates before rupture. The full thinning process can be approximated as a succession of two or three linear regimes. From the slope of the initial linear regime and assuming that this linear
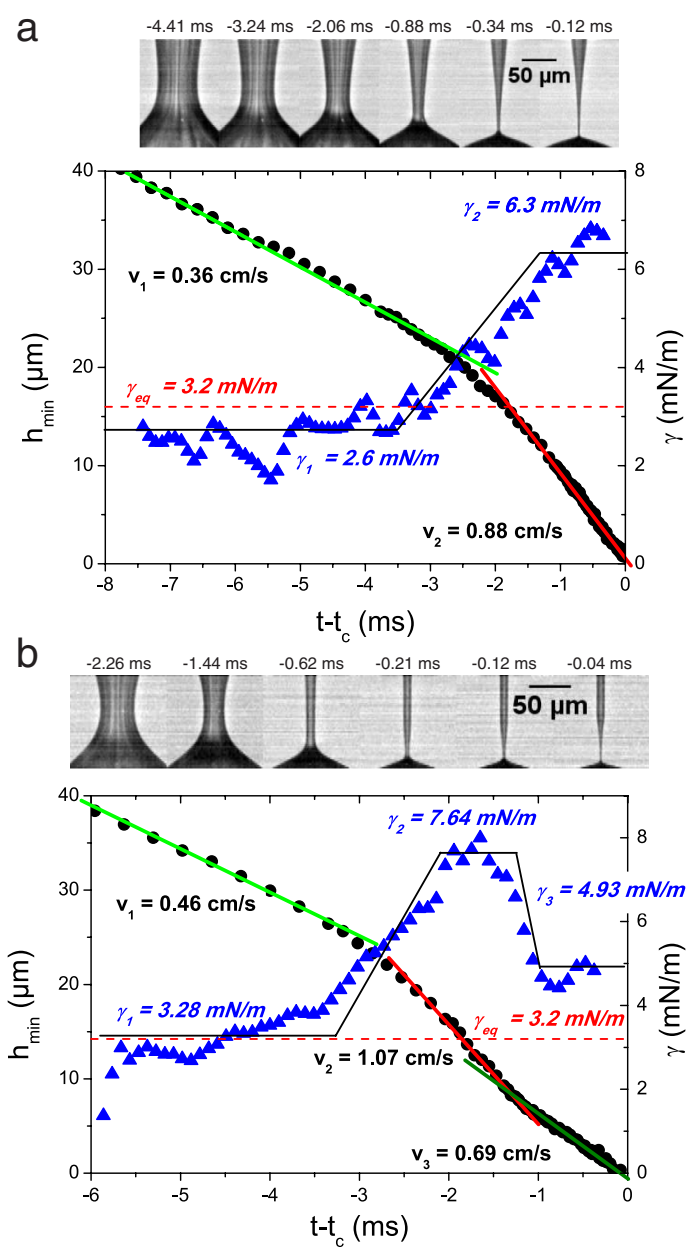

FIG. 2 (color online). Minimum neck radius (closed circles) and surface tension (solid triangles), calculated from a local derivative of the thinning curves, versus time for a LC drop pinching in a $2 \mathrm{cmc}$ water SDS solution. The injection flux of the LC is $20 \mu \mathrm{l} / \mathrm{h}$ that of the water solution is $5 \mathrm{ml} / \mathrm{h}$ in (a) and $2 \mathrm{ml} / \mathrm{h}$ in (b). Solid horizontal lines: surface tension extracted from a linear fit to each regime. Dashed lines: equilibrium surface tension. The viscous length scale is $2 \mathrm{~mm}$. Photographs of the rupture are shown for each case.

regime is consistent with the thinning law given above, we extract a surface tension $\gamma$ in good agreement with the measured equilibrium surface tension, $\gamma_{\mathrm{eq}}=3.2 \mathrm{mN} / \mathrm{m}$ obtained from measurements of the contact angle of LC drops on the SDS solution and from pendant drop experiments. A similar conclusion was drawn from a study of different surfactant concentrations for which the first linear part of the thinning curves gives a surface tension in good agreement with the ones obtained from our pendant drop measurements. These measurements give a surface tension of $12.5,6,4,3.2$ and $2.7 \mathrm{mN} / \mathrm{m}$ for concentrations of 0.1 , $0.5,0.8,2$, and $5 \mathrm{cmc}$, respectively. These values are to be compared to the ones extracted from the first linear regime which gives a surface tension of $13 \pm 2,7 \pm 1,3.7 \pm 0.4$, $3 \pm 0.4$, and $2.4 \pm 0.4 \mathrm{mN} / \mathrm{m}$. The second linear regime is steeper and the extracted surface tension is correspondingly higher. 
According to recent numerical simulations [6,8], the surfactant concentration should be lower in the region of the rapidly thinning neck so that the surface tension increases in time. If we extract the surface tension from the local slope of the full thinning curve, we obtain a time dependent surface tension as shown in Fig. 2. The surface tension starts out at values close to its equilibrium value and increases as the transition from the first linear regime to the second linear regime is approached. The surface tension then reaches a new roughly constant value in the second linear regime. This observation is in line with depletion of surfactant at the location of the minimum neck radius despite the relatively large surfactant concentration used. Measurements using the Maximum Bubble Pressure Method for the aqueous SDS-air interface at $2 \mathrm{cmc}$ give an exponential dependence of the dynamic surface tension versus time with a time constant of $72 \pm$ $5 \mathrm{~ms}$. This agrees with previous dynamic surface tension measurements [21] which find a time constant of order $100 \mathrm{~ms}$ for the surface to be replenished with surfactant. The time scales in the pinch-off experiments being of order a millisecond and therefore much smaller than the characteristic time for replenishing the surface, depletion of surfactant may occur in accordance with our observations. The effects due to the presence of surfactant were observed for concentrations ranging from $0.1 \mathrm{cmc}$ up to $5 \mathrm{cmc}$ indicating that surfactant depletion may occur both below and above the cmc.

In the case of 3 linear regimes, a third surface tension plateau is observed for the last instants. The difference between the two situations can also be observed in the displacement of the position of the minimum neck radius $z_{h_{\min }}(t)$, defined as the distance to the snap off position, as shown in Fig. 3. An examination of the pinching region, shows that $z_{h_{\min }}(t)$ changes continuously for Fig. 2(a) with the profile becoming more and more conical as expected for two-fluid pinch-off. However, for the three regime case, the minimum location changes slowly at first and as the third regime is approached, the neck becomes cylindrical before a rapid displacement of the minimum neck radius towards a location near the drop. Only for this third regime do the profiles become conical as illustrated in the photographs of Fig. 2 and 3. A probable reason for the smaller slope and therefore the smaller value of the surface tension for the third linear regime is that at this new location near the drop, the concentration of surfactant is higher. In previous numerical work [6,8], the concentration of surfactant at the interface does go through a minimum at the pinch location. Additional insight is also gained from carefully observing the thinning curves. We have noted that before the onset of the third linear regime, the thinning dynamics slows down and the neck becomes cylindrical giving a very shallow minimum [see photos in Figs. 2(b) and 3]. It is only after this slowing down, which was more or less pronounced depending on the flow rate and the concentration, that the minimum moves rapidly towards

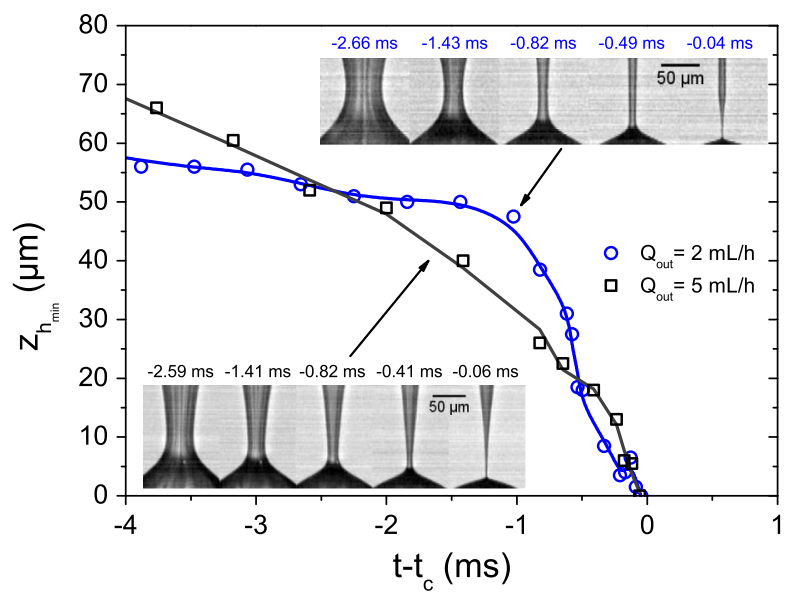

FIG. 3 (color online). Evolution of $z_{h_{\min }}$ for the ruptures shown in Fig. 2. Photographs of the rupture region are shown for the two situations.

the drop and the thinning resumes to give rise to the third linear regime. It has been suggested [6] that the depletion of surfactant from the minimum neck location may generate Marangoni stresses: This depletion creates a surface tension gradient between the minimum location, where the surface tension would be large, and the outer regions where the surface tension would be smaller, forcing liquid to move towards the minimum location. This should slow down the dynamics as we observe. Note that this slowing down is absent in Fig. 2(a) where the neck remains conical all through the second linear regime with the minimum continuously moving towards its final location. Conical profiles are expected for two-fluid pinch-off $[18,19]$ but the slopes measured here are smaller than expected, probably due to surface tension gradients.

Further evidence of depletion for fluid pinch-off in the presence of surfactants comes from the second experiment which considers the detachment of a drop that slowly emerges from a $2 \mathrm{~mm}$ nozzle. These additional experiments using a Newtonian fluid in a simpler set up allow us to check for the generality of the depletion observed above. We study the rupture of high viscosity water-glycerol mixtures with and without the presence of the surfactant SDS. Without surfactant and for the liquid viscosities used here, the thinning of the neck has been shown to proceed in two different but linear stages. Both these scalings exist below the viscous length scale $l_{\nu}=\frac{\eta^{2}}{\rho \gamma}$ where $\rho$ is the fluid density. The first stage was predicted by Papageourgiou [22] for the case where the Reynolds number is negligible and is known as visco-capillary break up. This prediction reads $h_{\min }(t)=0.07 \frac{\gamma}{\eta}\left(t-t_{c}\right)$. For later stages, the velocity of the fluid at the neck increases and the assumption of negligible Reynolds number is no longer valid. A new regime was predicted by Eggers [23], known as viscocapillary-inertial break up, and the resulting thinning law reads: $h_{\min }(t)=0.03 \frac{\gamma}{\eta}\left(t-t_{c}\right)$. The thinning dynamics in this case has been studied experimentally [24] and the 

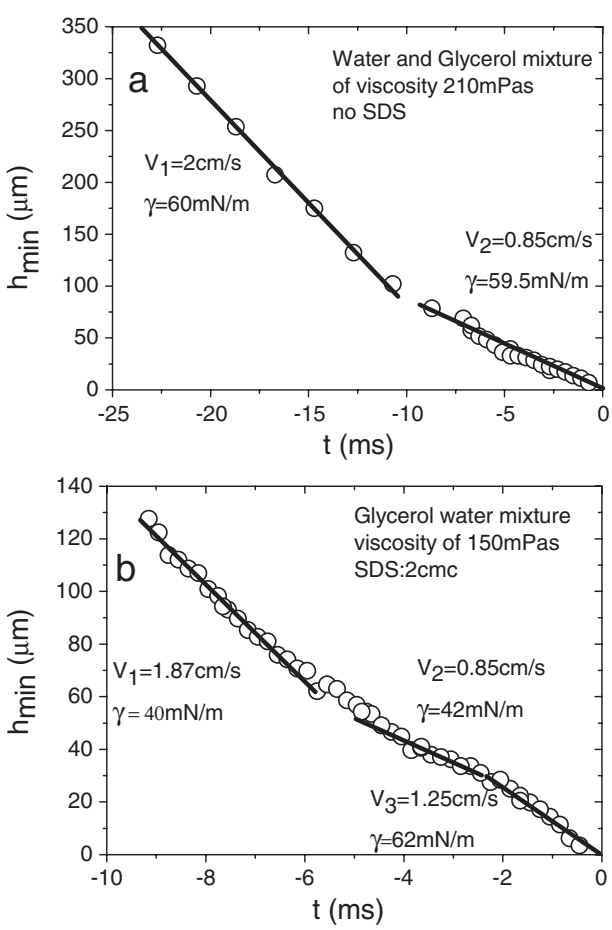

FIG. 4. Minimum neck radius versus time: (a) $210 \mathrm{mPa} \cdot \mathrm{s}$ water-glycerol mixture (b) $150 \mathrm{mPa} \cdot \mathrm{s}$ water-glycerol mixture in the presence of SDS $(2 \mathrm{cmc})$. The equilibrium surface tension is $60 \mathrm{mN} / \mathrm{m}$ and $40 \mathrm{mN} / \mathrm{m}$ in the two cases, respectively. The surface tension extracted from the slopes of the first two regimes are indicated. The third regime in (b) has a surface tension comparable to that of the bare interface. The viscous length scale for these two solutions is 400 and $460 \mu \mathrm{m}$.

transition from the first to the second regime has been identified. Our experiments are carried out under closely similar conditions as in [24] and we indeed observe the presence of two linear regimes as shown in Fig. 4(a). The surface tension values extracted from the slopes of the first regime and the second regime, using the predicted slopes quoted above, are in excellent agreement with the measured value of $60 \mathrm{mN} / \mathrm{m}$.

In the presence of surfactant (at $2 \mathrm{cmc}$ ), this scenario barely changes at its early instants as shown in Fig. 4(b). Again, two regimes with different slopes appear. The only difference is the value of the surface tension which is that of the surfactant covered interface. However, during the second stage of the thinning (the Eggers regime), an increase in slope is observed giving rise to a third regime. This increase of the slope is indicative of a change in surface tension. This steepening therefore heralds a depletion of surfactant at the interface with the ensuing increase in rupture speed. The surface tension for this last stage $(62 \mathrm{mN} / \mathrm{m})$ is close to that of the bare solution: most of the surfactant has been depleted from the pinch region. This differs from the previous case where not all of the surfactant was depleted from the pinch region for a similar surfactant concentration. The difference stems from the fact that in the first case a reservoir of surfactant is present in the low viscosity surrounding fluid while in the second case, the surfactant is present in the rupturing fluid with a large viscosity. The diffusion of surfactant from the bulk to the surface is therefore very different in the two cases showing that for small diffusivities, the surfactant may be depleted in full while for the high diffusivity case, surfactant from the surrounding phase may relieve some of the depletion. For the first situation presented above, almost full depletion was achieved only for the lowest concentration $(0.1 \mathrm{cmc})$ examined.

The presence of surface active agents at the surface of a rupturing fluid neck affects the rupture process in different ways. Our results show this phenomenon in two ways: either through an increase of surface tension in the same location or a sudden decrease of the surface tension as the rupture location moves rapidly towards regions with higher concentrations. Our results also indicate that the surface remains partially covered with surfactant when diffusion from the bulk is sufficiently important. However, for slow diffusion, complete depletion may occur.

[1] J. Eggers and E. Villermaux, Rep. Prog. Phys. 71, 036601 (2008).

[2] J. Eggers, Rev. Mod. Phys. 69, 865 (1997).

[3] M-L. E. Timmermans and J. R. Lister, J. Fluid Mech. 459, 289 (2002).

[4] B. Ambravaneswaran and O. A. Basaran, Phys. Fluids 11, 997 (1999).

[5] Y. C. Liao, E. I. Franses, and O. A. Basaran, Phys. Fluids 18, 022101 (2006).

[6] P. T. McGough and O. A. Basaran, Phys. Rev. Lett. 96, 054502 (2006).

[7] Q. Xu, Y. C. Liao, and O. A. Basaran, Phys. Rev. Lett. 98, 054503 (2007).

[8] F. Jin, N. R. Gupta, and K. J. Stebe, Phys. Fluids 18, 022103 (2006).

[9] S. L. Anna, N. Bontoux, and H. A. Stone, Appl. Phys. Lett. 82, 364 (2003).

[10] P. K. Rai, M. M. Denn, and C. Maldarelli, Langmuir 19, 7370 (2003).

[11] P. S. Drzaic, Liquid Crystal Dispersions (World Scientific, Singapore, 1995).

[12] D. Rudhardt et al., Appl. Phys. Lett. 82, 2610 (2003).

[13] K. Skarp, S. T. Lagerwall, and B. Stebler, Mol. Cryst. Liq. Cryst. 60, 215 (1980).

[14] A. G. Chmielewski, Mol. Cryst. Liq. Cryst. 132, 339 (1986).

[15] K. Negita, J. Chem. Phys. 105, 7837 (1996).

[16] M. H. Wu et al., J. Soc. Rheol., Jpn. 31, 91 (2003).

[17] J. R. Lister and H. A. Stone, Phys. Fluids 10, 2758 (1998).

[18] I. Cohen et al., Phys. Rev. Lett. 83, 1147 (1999).

[19] W.W. Zhang and J. R. Lister, Phys. Rev. Lett. 83, 1151 (1999).

[20] I. Cohen and S. R. Nagel, Phys. Fluids 13, 3533 (2001).

[21] N. C. Christov et al., Langmuir 22, 7528 (2006).

[22] D. T. Papageourgiou, Phys. Fluids 7, 1529 (1995).

[23] J. Eggers, Phys. Rev. Lett. 71, 3458 (1993).

[24] A. Rothert, R. Richter, and I. Rehberg, Phys. Rev. Lett. 87, 084501 (2001). 